\title{
Malignant fungal infection of the cavernous sinus: case report
}

\author{
Jayson A. Neil, MD, ${ }^{1}$ Richard R. Orlandi, MD, ${ }^{2}$ and William T. Couldwell, MD, PhD' \\ 'Department of Neurosurgery; and 2Department of Surgery, Division of Otolaryngology, University of Utah School of Medicine, \\ Salt Lake City, Utah \\ Intracranial spread of fungal infection is a life-threatening condition that usually affects immunocompromised patients. \\ Here the authors present a case of biopsy-proven Aspergillus fumigatus infection of the paranasal sinuses in an immu- \\ nocompetent patient with documented spread to the orbit, cavernous sinus, and petrous apex despite medical antifungal \\ treatment. As a life-saving treatment, cavernous sinus resection with external carotid artery-middle cerebral artery \\ bypass was performed. The authors discuss the literature regarding the intracranial spread of paranasal sinus fungal \\ infections in immunocompetent patients and management strategies.
}

http://thejns.org/doi/abs/10.3171/2015.2.JNS142668

KEY WORDS cavernous sinus resection; Aspergillus fumigatus; paranasal fungal infection; cavernous sinus infection; ECA-MCA bypass

$\mathrm{U}$ NIVERSALLY present in soil, water, and air, Aspergillus is a spore-forming fungus to which immunocompetent individuals are typically less susceptible. Aspergillosis may present in a noninvasive form such as allergic rhinitis, a fungal ball with local invasion, or even hematological embolization. ${ }^{89}$ Intracranial spread of Aspergillus infection is uncommon, although there are documented cases of orbital Aspergillus infections even in immunocompetent patients. ${ }^{89}$ Direct contiguous spread can pose significant diagnostic difficulty not only because the disease is rare, but also because there is no specific imaging pattern. The disease can mimic other conditions including neoplasms, bacterial infections, optic neuropathy, and idiopathic inflammatory diseases. Despite the difficulty in diagnosis, aggressive management of these infections is required because the mortality rate can be as high as $75 \%$. $^{2,6,10}$ We report an extremely rare case of $A s-$ pergillus infection in an immunocompetent patient with direct contiguous spread from the paranasal sinus to the orbit, cavernous sinus, and petrous apex while the patient was on antifungal medication.

\section{Case Report \\ Clinical Presentation}

A 69-year-old farmer had experienced right eye vision loss of 2 months' duration that resulted in complete blindness 1 month prior to presentation. The patient believed this was due to a cataract, but an initial CT scan of the sinuses at an outside facility showed an opacified right anterior clinoid process with focal dehiscence along the optic canal (Fig. 1A). Magnetic resonance imaging with and without contrast enhancement demonstrated slight T2 hypointensity and enhancement along the orbital apex and optic canal (Fig. 1B-D).

Because of these imaging findings coupled with the rapidly progressive vision loss, the patient was referred to our institution, where he underwent endoscopic right ethmoidectomy, sphenoidotomy, decompression of the optic nerve, and biopsy of the lesion. Postoperatively, the patient showed no clinical improvement, and the pathological analysis was negative for tumor, bacteria, fungus, or mycobacteria. He had initially been started on steroids to reduce

ABBREVIATIONS CCA = common carotid artery; ECA = external carotid artery; ICA = internal carotid artery; $M C A=$ middle cerebral artery.

SUBMITTED November 24, 2014. ACCEPTED February 25, 2015.

INCLUDE WHEN CITING Published online August 28, 2015; DOI: 10.3171/2015.2.JNS142668. 

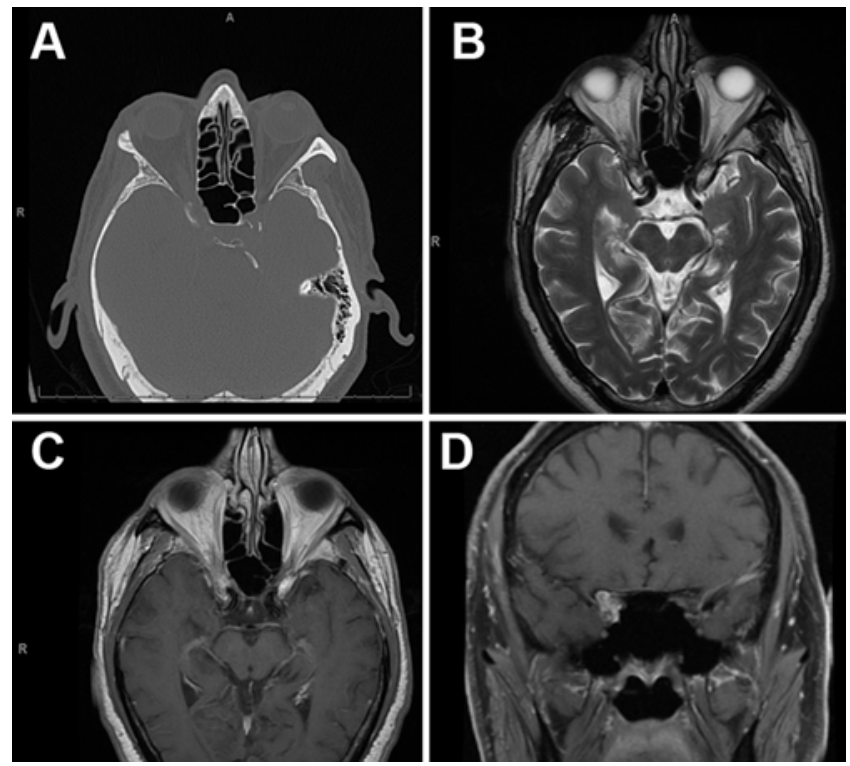

FIG. 1. Axial CT (A) demonstrating subtle evidence of an osteolytic lesion at the right orbital apex. Axial T2-weighted MR image (B) demonstrating a hyperdense lesion at the right orbital apex. Axial (C) and coronal (D) T1-weighted postcontrast MR images demonstrating a right orbital lesion.

inflammation, but he was weaned from these when there was no response, and he was monitored clinically with a plan for repeat imaging.

\section{Diagnosis and Preoperative Course}

When the patient began to have worsening headaches 2 months later, follow-up MRI with and without contrast revealed increasing enhancement along the orbital apex and optic canal, indicating progression of the lesion. At
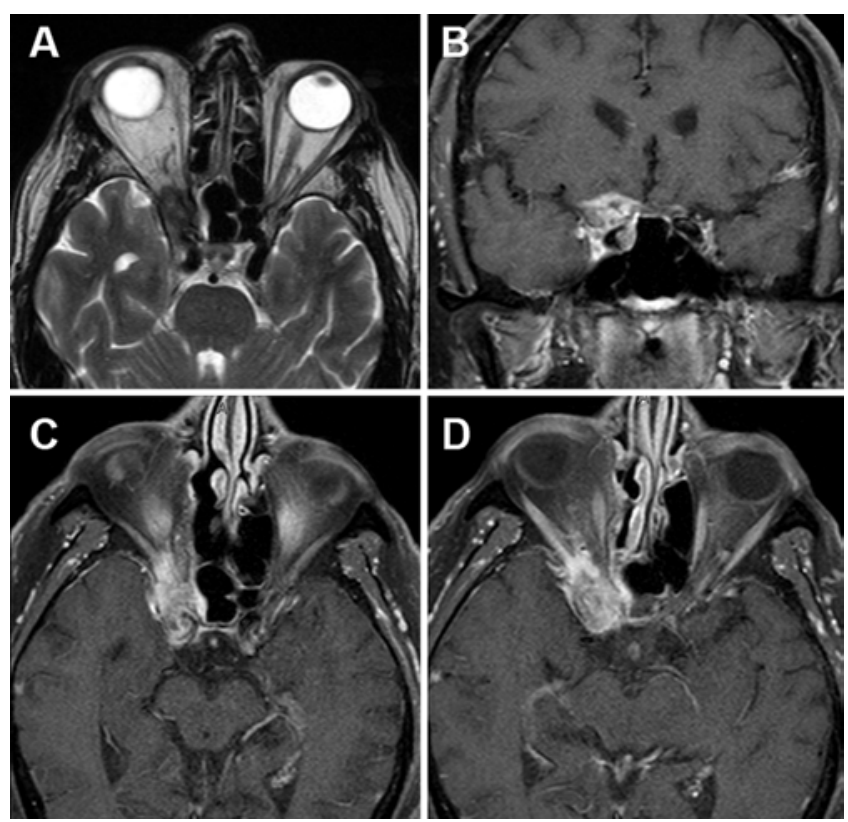

FIG. 2. Axial T2-weighted MR image (A) and coronal (B) and axial (C and D) T1-weighted fat-saturated postcontrast MR images demonstrating progression of a T2 hypointense, contrast-enhancing right orbital apex and cavernous sinus lesion. that time, further exploration and debridement was performed endoscopically. The pathological specimens from this surgery demonstrated fungal hyphae, and the patient was initially started on amphotericin lipid complex. The cultures speciated to Aspergillus fumigatus, which is the most common species of Aspergillus causing infection, ${ }^{8}$ and the patient's therapy was tailored to voriconazole.

He continued to have headaches with no improvement in vision, and repeat MRI performed 6 months later demonstrated increasing enhancement and progression of his infection into the orbit and cavernous sinus (Fig. 2). A preoperative cerebral angiogram demonstrated bilateral proximal cervical internal carotid artery (ICA) stenosis and right intracranial ophthalmic segment stenosis of 50\% (Figs. 3 and 4). Furthermore, balloon occlusion testing of the right common carotid artery (CCA) resulted in progressive left-sided weakness.

\section{Operation and Pathological Findings}

Given progression of the disease despite medical therapy, the patient underwent a right frontotemporal craniotomy for complete resection of the right cavernous sinus infection, external carotid artery (ECA)-middle cerebral artery (MCA) saphenous vein bypass, exenteration of the right orbit, harvest of abdominal fat graft for packing of the sphenoid sinus and orbit, and placement of a right frontal external ventricular drain. This was performed using a method similar to our previously published technique. ${ }^{3}$

An operative video demonstrates key portions of this case (Video 1).

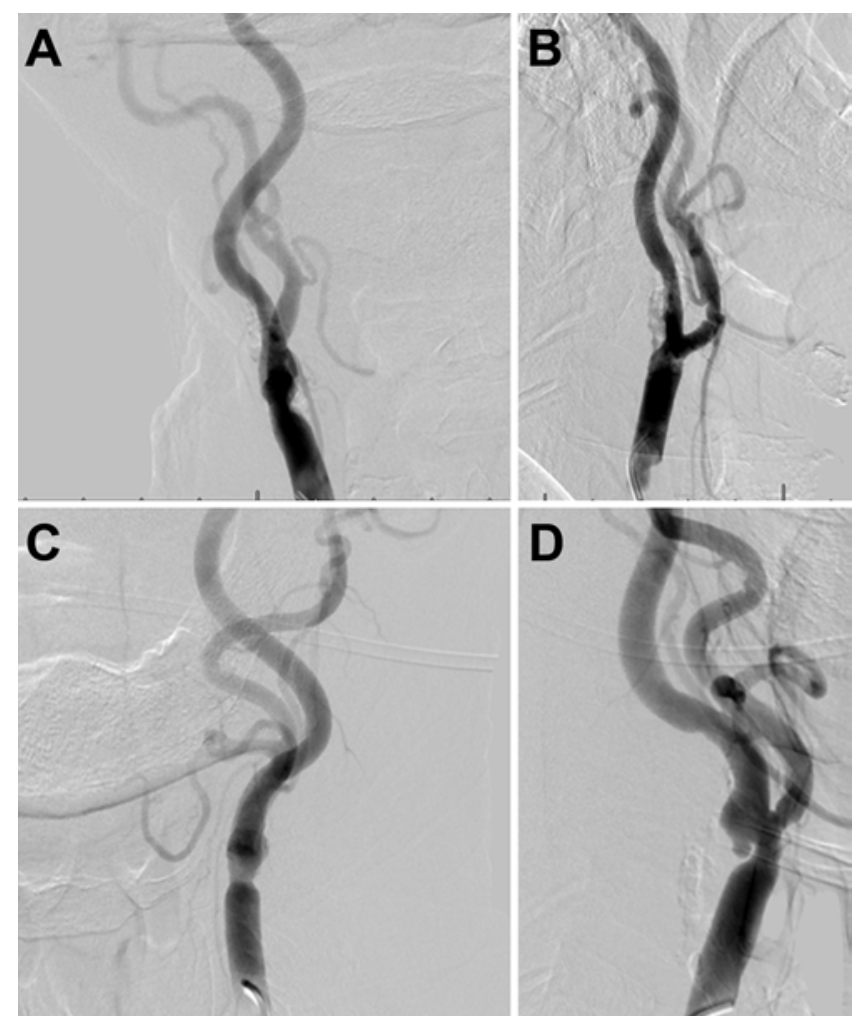

FIG. 3. Right (A and B) and left (C and D) CCA injections, anteroposterior ( $A$ and $C$ ) and lateral (B and $D)$ views, demonstrating ICA stenosis. 


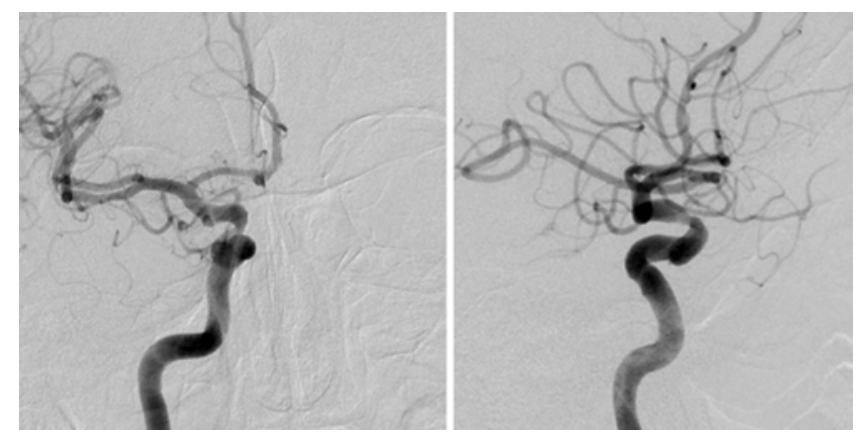

FIG. 4. Cerebral angiograms of the right ICA injection, anteroposterior (left) and lateral (right) views, demonstrating ophthalmic segment stenosis.

VIDEO 1. Operative video demonstrating key portions of the cavernous sinus resection with ECA-MCA bypass. This procedure was used to treat a case of biopsy-proven Aspergillus infection of the paranasal sinuses in an immunocompetent patient with direct contiguous spread despite antifungal medication. Copyright Vance Mortimer. Published with permission. Click here to view with Media Player. Click here to view with Quicktime.

The patient was placed supine on the operating table with a right shoulder roll. A Mayfield 3-pin head clamp was placed with the head turned approximately $30^{\circ}$ to the left with some slight extension. The skin was marked for right frontotemporal, right neck, abdomen, and right leg incisions (Fig. 5A and B). A frontotemporal craniotomy was performed, and the sphenoid wing and temporal fossa were drilled flush. The right neck was incised, the CCA, ICA, and ECA were exposed and isolated, and the hypoglossal nerve was protected with a vessel loop. The middle fossa floor anatomy was explored, the foramen spinosum was located, and the middle meningeal artery was coagulated and cut. A cutting bit and a diamond bit were used to drill a hole in the middle fossa, just lateral to the foramen ovale, for a submandibular approach for the saphenous vein graft. The dura mater was opened, the sylvian fissure was split, and the optic nerve, carotid artery, MCA, and 2 large $\mathrm{M}_{2}$ branches were located.

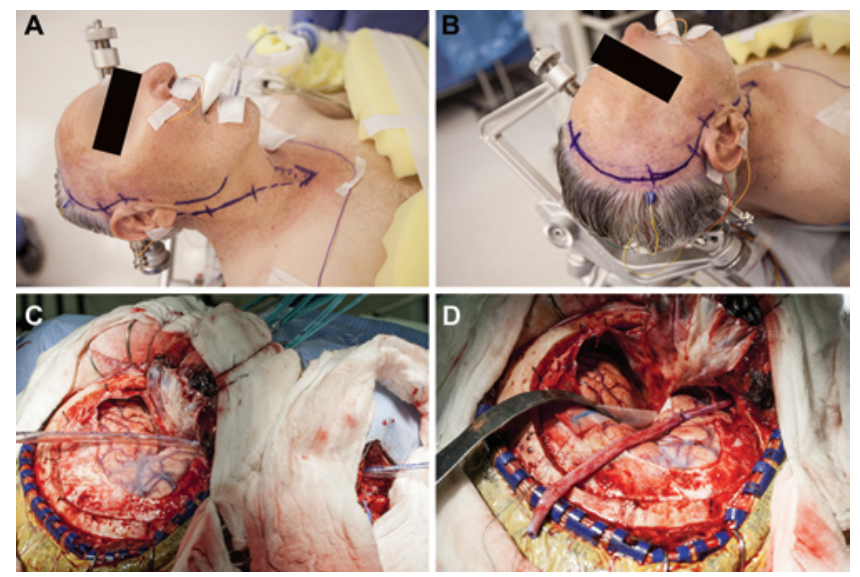

FIG. 5. Intraoperative photos demonstrating both patient positioning with incisions marked for the scalp and neck (A and B) and the use of a chest tube for tunneling the saphenous vein graft (C) with the graft exiting the middle fossa skull base tunnel (D). Figure is available in color online only.
The saphenous vein graft was harvested endoscopically and anastomosed to the ECA using 7-0 Prolene sutures, then tunneled through the hole in the middle fossa floor (Fig. 5C and D), and anastomosed to the $\mathrm{M}_{2}$ branch using 9-0 nylon sutures. The proximal carotid artery was clamped, and flow through the graft was checked with direct visual inspection, Doppler ultrasonography, and indocyanine green. The proximal cervical ICA was then ligated and cut. The same was performed just proximally to the intracranial carotid bifurcation.

For cavernous sinus resection, an anterior cut was made along the superior and lateral orbital roof and extended laterally through the foramina rotundum and ovale and then posteriorly to the petrous apex and medially to the tentorium, with care to avoid any injury to the cochlea. The superior petrosal sinus was packed. Anteriorly along this course, cranial nerves II, III, IV, V1, V2, and VI were encountered along their distal ends and divided beyond any infection. Posteriorly, cranial nerves II, IV, V, and VI were encountered along their proximal end and likewise were sectioned beyond the extent of infection. The petrous carotid was dissected, ligated, and divided. A medial cut was performed from the anterior medial edge of the superior orbital cut posteriorly straight back and into the sphenoid sinus, which was carried around the lateral edge of the sella to avoid injury to the pituitary. The cut was then extended from the lateral edge of the sella posteriorly to the posterior clinoid and then across the dura of the clivus.

At surgery, the cavernous sinus, posterior orbital contents, and petrous apex were resected in an en bloc manner. Of note, there was no evidence of thickened arachnoid indicating basilar meningitis throughout the region. Pathological analysis again demonstrated A. fumigatus (Fig. 6). The right orbit was enucleated to avoid infection and sympathetic ophthalmia. The wound was packed with autologous fat graft and sealed with a pericranial flap and fibrin glue. A nasoseptal flap was created to close the sphenoid sinus, and a right frontal external ventricular drain was placed. The dura was closed with sutured dural substitute, the bone flap was replaced with titanium plates and screws, and bony defects were covered with Medpor. The wounds were then closed in a standard fashion.

\section{Postoperative Course}

The patient had no unexpected postoperative neurological defects given sectioning of the above mentioned cranial nerves. Head CT, CT angiography, and cerebral angiography demonstrated expected postoperative findings and a

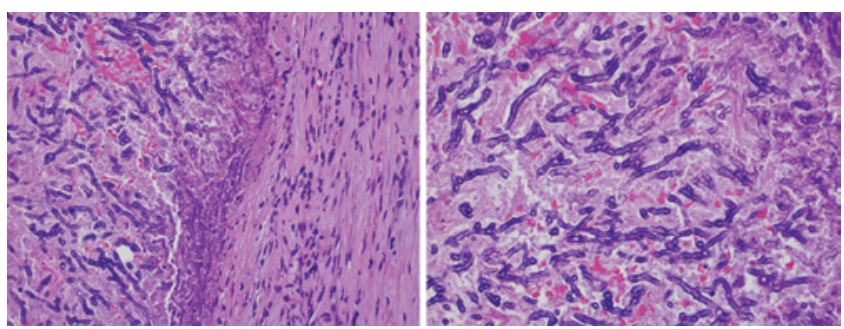

FIG. 6. Photomicrographs demonstrating fungal hyphae consistent with Aspergillus. $\mathrm{H} \& \mathrm{E}$, original magnification $\times 40$ (left) and $\times 60$ (right). Figure is available in color online only. 


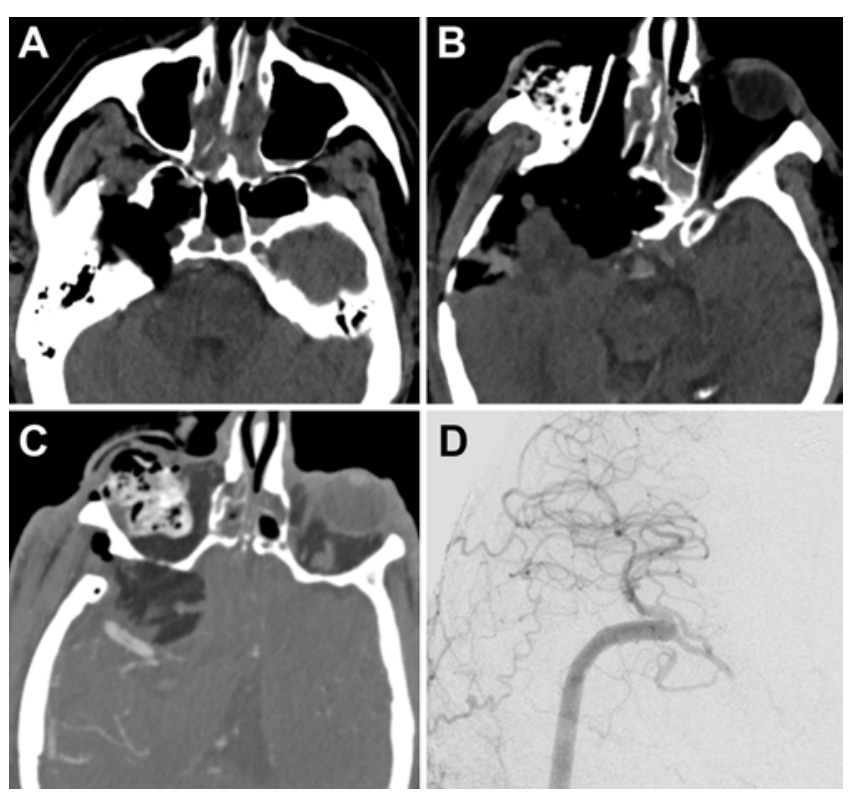

FIG. 7. Axial CT scans (A and B) demonstrating expected postoperative findings for right cavernous sinus and petrous apex resection. Axial CT angiogram (C) demonstrating ECA-MCA bypass. Anteroposterior cerebral angiogram (D) showing patent saphenous ECA-MCA bypass.

patent ECA-MCA saphenous vein graft (Fig. 7); however, the postoperative course was complicated by pneumonia requiring prolonged intubation, pulmonary effusion requiring a chest tube, and deep vein thrombosis requiring heparin drip. Over the following 3 weeks, the patient was extubated, the chest tube was removed, and the patient was weaned from the external ventricular drain prior to transfer to a rehabilitation unit. He continued to take aspirin, warfarin, and a prolonged course of voriconazole. Six months after resection, he remained free of demonstrable disease (Fig. 8).

\section{Discussion}

Although invasive Aspergillus infection is more common in immunocompromised individuals, there are reports in immunocompetent patients as well. ${ }^{8}$ The presentation tends to be chronic and indolent, with pain being the most consistent complaint. Although the risk of $A s$ pergillus infection can be increased by the use of steroids, antineoplastic or immunosuppressive medications, and alcohol or illicit drugs, none of these were initially present in our patient.

\section{Diagnosis}

Diagnosis of these lesions is often delayed, particularly because the interpretation of imaging can be difficult. The most common CT imaging features include hyperdense lesions with intracranial extension and bone destruction. On MRI, there are areas of hypointense signal on T2weighted sequences with areas of restricted diffusion, decreased perfusion, and hemorrhage. ${ }^{9}$ Biopsy is required to establish the presumptive radiological diagnosis.

\section{Medical and Surgical Treatment}

Once the infection is identified, aggressive manage-
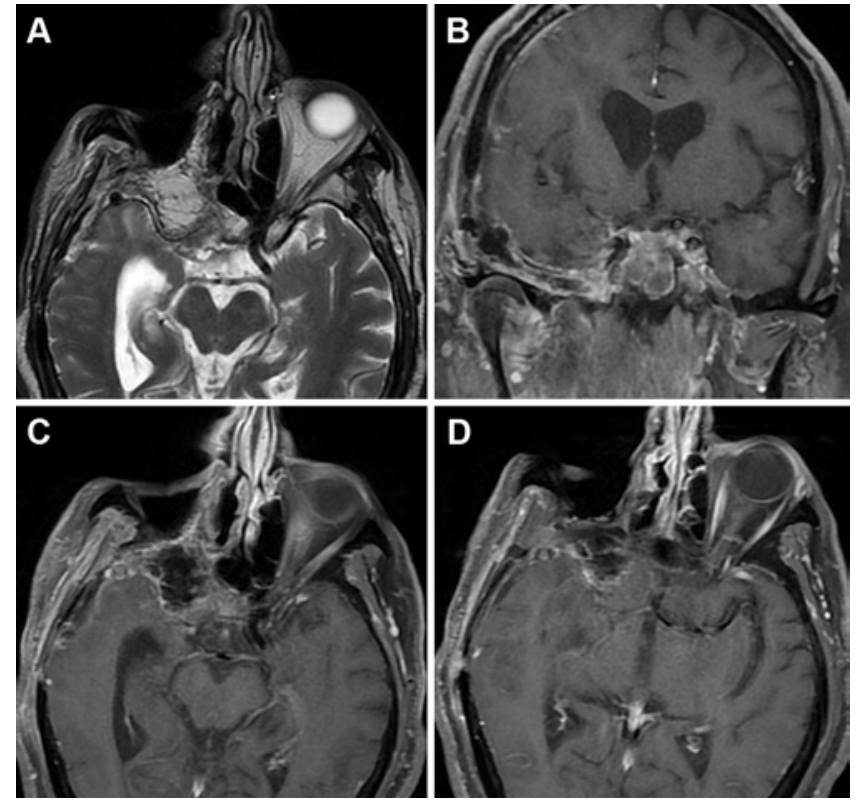

FIG. 8. Axial T2-weighted (A) MR image and coronal (B) and axial (C and D) T1-weighted fat-saturated postcontrast MR images obtained 6 months after surgery, demonstrating no evidence of residual disease.

ment is necessary. Unfortunately, the response rate to medical therapy is only $40 \%-60 \%$. $^{2,6}$ The mortality rate of invasive sino-orbital aspergillosis is also $40 \%-75 \%$ despite maximal management including surgical debridement and antifungal therapy., $2,6,10$

Our patient was initially treated with antifungals including amphotericin and voriconazole, but the infection progressed despite these therapies. We chose to proceed with surgical debridement, which in this case required resection of the cavernous sinus and a portion of the petrous apex. We have previously described the operative technique and noted that cavernous sinus resection carries significant morbidity and a mortality rate that can approach $25 \% .^{3}$ Indications for cavernous sinus resection are rare and poorly defined; progressive fungal infection despite treatment may represent one of them.

The decision to proceed with cavernous sinus resection should be approached with marked caution and deliberation. This deliberation should include planning to address the cranial nerves, the carotid artery, and reconstruction of the skull base defect. For complete unilateral cavernous sinus resection, cranial nerves II-VI will be resected. In such cases, a new cranial nerve deficit is not a major consideration; for example, in the present case the patient had preoperative blindness and ophthalmoplegia in the affected eye. In cases of infection, care should be employed to ensure that all infected tissue is removed, especially if the infection involves proximal or distal cranial nerve tissue (for example, the root entry zone or beyond the foramen rotundum or ovale with resection of cranial nerve $\mathrm{V}$ ).

Similarly, management of the carotid artery should be planned in detail prior to resection. In general, carotid artery sacrifice will be necessary for cavernous sinus resection based on the pathology. Proceeding with revascularization is controversial. We use a selective approach including balloon occlusion testing, as in this patient 
and as previously described. ${ }^{11}$ There is limited literature regarding the risk of bypass specific to carotid sacrifice for skull base lesions, but the published rates of morbidity are 3\%-20\% and mortality rates of less than $6 \%$ have been reported. ${ }^{1,4,5,7}$ These risks should be weighed against the risk of carotid sacrifice in skull base lesions without bypass, for which there is neurological morbidity of $17 \%$ and mortality of $7 \%$. In the present case, we chose to perform an ECA-MCA bypass. An end-to-side ECA origin allows brain perfusion during the proximal anastomosis. The MCA distal site ensures an anastomosis remote from the infection. Previous experience with bypass grafts in patients with fungal infections with arterial involvement has indicated that anastomosis sites even a short distance from the infection may result in higher thrombosis rates. ${ }^{3}$

Reconstruction of the skull base should be carefully planned. In the featured case, there were openings into the sphenoid sinus, the pterygopalatine fossa, and the infratemporal fossa. We prefer to use autologous tissue whenever possible, and vascularized tissue is ideal. All defects were covered with autologous fat and pericranium grafts. The opening in the sphenoid sinus can also be closed externally with a nasal-septal flap. Because of the risk of cerebrospinal fluid leakage, dural closure must be meticulous, and cerebrospinal fluid diversion is recommended. In cases with larger defects, vascularized flaps, including free flap or pedicled temporalis flap, should be considered.

\section{Conclusions}

Invasive sino-orbital Aspergillus infection that progresses to infect the cavernous sinus despite medical therapy carries a high mortality risk and may be an indication for cavernous sinus resection. Even when this operation is performed with extensive preoperative planning by experienced surgeons at quaternary medical centers, the morbidity is significant, but it may outweigh the risk of morbidity and mortality to the patient given the natural history of this disease.

\section{Acknowledgments}

We thank Kristin Kraus, MSc, for editorial assistance with this paper, and Vance Mortimer for editorial assistance with the attached video.

\section{References}

1. Abdulrauf SI: Extracranial-to-intracranial bypass using radial artery grafting for complex skull base tumors: technical note. Skull Base 15:207-213, 2005

2. Choi HS, Choi JY, Yoon JS, Kim SJ, Lee SY: Clinical characteristics and prognosis of orbital invasive aspergillosis. Ophthal Plast Reconstr Surg 24:454-459, 2008

3. Couldwell WT, Macdonald JD, Taussky P: Complete resection of the cavernous sinus-indications and technique. World Neurosurg 82:1264-1270, 2013

4. Kalani MY, Kalb S, Martirosyan NL, Lettieri SC, Spetzler
RF, Porter RW, et al: Cerebral revascularization and carotid artery resection at the skull base for treatment of advanced head and neck malignancies. J Neurosurg 118:637-642, 2013

5. Lawton MT, Spetzler RF: Internal carotid artery sacrifice for radical resection of skull base tumors. Skull Base Surg 6:119-123, 1996

6. Mauriello JA Jr, Yepez N, Mostafavi R, Barofsky J, Kapila $\mathrm{R}$, Baredes S, et al: Invasive rhinosino-orbital aspergillosis with precipitous visual loss. Can J Ophthalmol 30:124-130, 1995

7. Mendelowitsch A, Taussky P, Rem JA, Gratzl O: Clinical outcome of standard extracranial-intracranial bypass surgery in patients with symptomatic atherosclerotic occlusion of the internal carotid artery. Acta Neurochir (Wien) 146:95-101, 2004

8. Mody KH, Ali MJ, Vemuganti GK, Nalamada S, Naik MN, Honavar SG: Orbital aspergillosis in immunocompetent patients. Br J Ophthalmol 98:1379-1384, 2014

9. Saini J, Gupta AK, Jolapara MB, Chatterjee S, Pendharkar HS, Kesavadas C, et al: Imaging findings in intracranial aspergillus infection in immunocompetent patients. World Neurosurg 74:661-670, 2010 (Erratum in World Neurosurg 78:e1, 2012)

10. Sivak-Callcott JA, Livesley N, Nugent RA, Rasmussen SL, Saeed P, Rootman J: Localised invasive sino-orbital aspergillosis: characteristic features. Br J Ophthalmol 88:681-687, 2004

11. Taussky P, Couldwell W: Decision-making strategies for ECIC bypass in the treatment of skull base tumors, in Abdelrauf SI (ed): Cerebral Revascularization: Techniques in Extracranial-to-Intracranial Bypass Surgery. Philadelphia: Saunders, 2010, pp 349-354

\section{Disclosure}

Dr. Orlandi is a consultant for Medtronic ENT.

\section{Author Contributions}

Conception and design: Couldwell. Acquisition of data: all authors. Analysis and interpretation of data: Neil, Orlandi. Drafting the article: Neil. Critically revising the article: Couldwell, Neil. Reviewed submitted version of manuscript: all authors. Approved the final version of the manuscript on behalf of all authors: Couldwell.

\section{Supplemental Information \\ Videos}

Video 1, Media Player. http://mfile.akamai.com/21490/wmv/ digitalwbc.download.akamai.com/21492/wm.digitalsource-naregional/jns14-2668_video_1.asx.

Video 1, Quicktime. http://mfile.akamai.com/21488/mov/ digitalwbc.download.akamai.com/21492/qt.digitalsource-global/ jns14-2668_video_1a.mov.

\section{Correspondence}

William T. Couldwell, Department of Neurosurgery, Clinical Neurosciences Center, University of Utah, 175 N. Medical Dr. E, Salt Lake City, UT 84132. email: william.couldwell@hsc.utah. edu. 